\title{
Photonic bandgaps in selectively filled photonic crystal fibers
}

\author{
Olga Jaworska*, Sławomir Ertman \\ Faculty of Physics, Warsaw University of Technology, Koszykowa 75, 00-662 Warszawa, Poland
}

Received August 23, 2017; accepted September 28, 2017; published September 30, 2017

\begin{abstract}
Simulations of selectively filled photonic crystal fibers using a finite elements method were performed. Different patterns of filling were modeled and compared to an empty and fully filled fiber. The dependence of effective refractive indices of guided modes, phase birefringence and confinement losses on guided wavelength was investigated. A comparison of the width of photonic bandgaps in different structures was made.
\end{abstract}

Photonic crystal fibers (PCFs) can be distinguished by the periodic structure of holes around a solid or hole fiber core. These holes can be filled with gas, liquid, or solid materials [1], giving rise to applications such as molecule detectors, temperature sensors, and all-in-fiber tunable polarizers and attenuators [2-3]. It is also possible to fill a certain part of the structure in a desired pattern to achieve further modification of fiber transmission parameters.

The applications of selectively filled PCFs include directional sensors made by filling the PCF structure asymmetrically with isotropic or anisotropic materials [45]. Hybrid and highly birefringent fibers can also be easily made by filling a selected pattern of holes [6].

The investigations of selectively filled PCFs are focused on experiments and modeling for certain applications, and so far little attention has been given to comparison of different structures and their optical properties.

In this work, we performed numerical modeling of selectively filled photonic crystal fibers using the finite elements method.

The modeled fiber structure has the following parameters:

- hole diameter $(d): 2.2 \mu \mathrm{m}$,

- hole spacing $(\Lambda): 4.4 \mu \mathrm{m}$,

- 5 "rings" of holes around core in hexagonal lattice,

- material: n=1.6 (i.e. Schott F2 glass).

The modeled filling material was isotropic with a refractive index of 1.7 (i.e. Cargille refractive index liquids "Series M"). The refractive index of the filling material is higher than the index of the glass because we decided to focus on band gap guiding in a selectively filled fibers.

We investigated selectively filled structures that were symmetrical with respect to two fiber axes. The modeled structures are shown in Fig. 1. Two structures can be considered as isotropic (empty fiber and fiber completely

*E-mail: olgaqsi@gmail.com filled with liquid - Fig. 1a,f). Four remaining structures (Fig. 1b-e) are birefringent due to geometry (similarly like e.g. in [7]). It is worth noticing that although the filling patterns are different, the geometry of the six holes closest to the core is the same for those four structures (Fig. 1b-e).

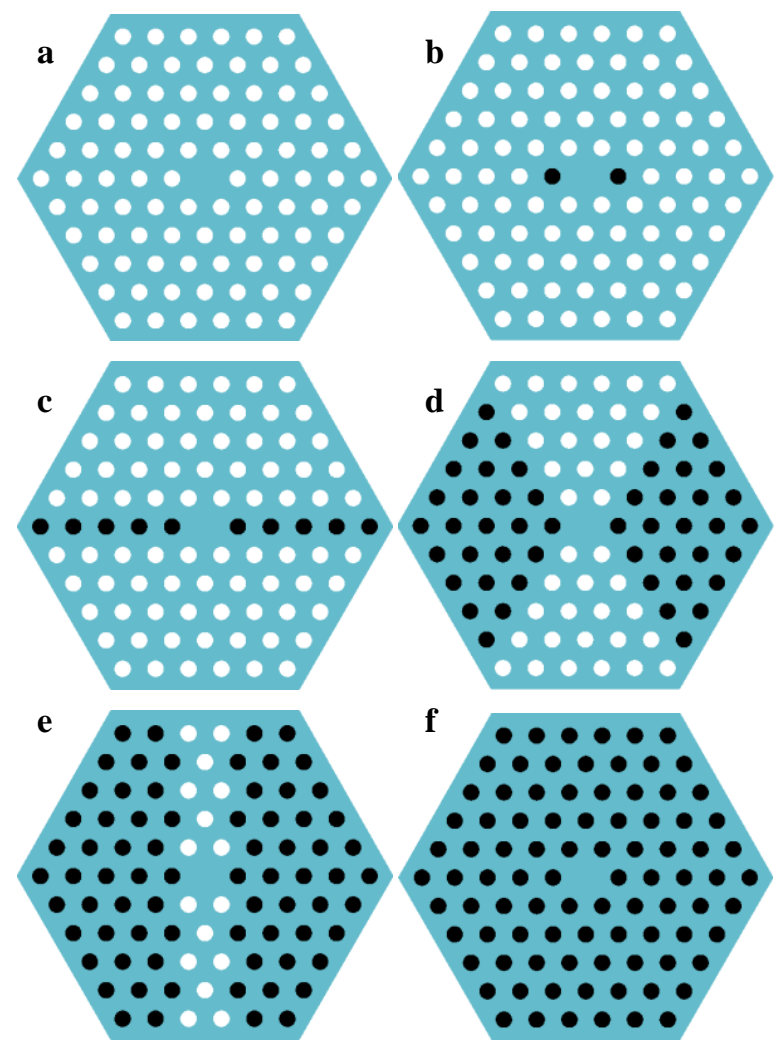

Fig. 1. Investigated patterns of PCF structures. Filled holes are shown in black.

Full vector finite element method calculations were performed using commercial software Comsol Multiphysics. Each structure was modeled in a wide spectral range (400-1800nm) and two orthogonal components of the fundamental mode were analyzed. Based on the modeled effective refractive indices of guided modes, dependences of the phase birefringence, band gap positions and confinement losses on the guided wavelength were investigated. 
In the empty structure, the refractive index of the core is larger than the effective refractive index of the cladding, therefore both polarizations are index-guided. In a selectively filled and fully filled structure both polarizations are guided by a photonic bandgap. It means that hybrid guiding was not observed.

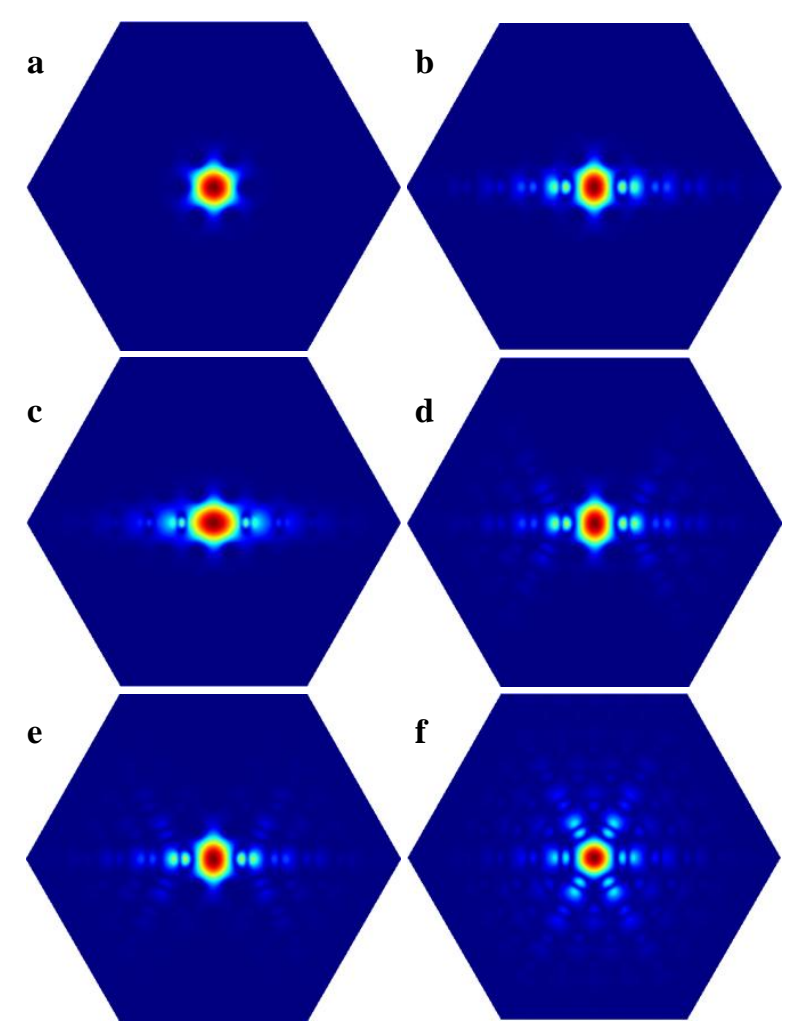

Fig. 2. Modal fields of x-polarization in investigated structures (exemplary images calculated for wavelength of 1550nm)

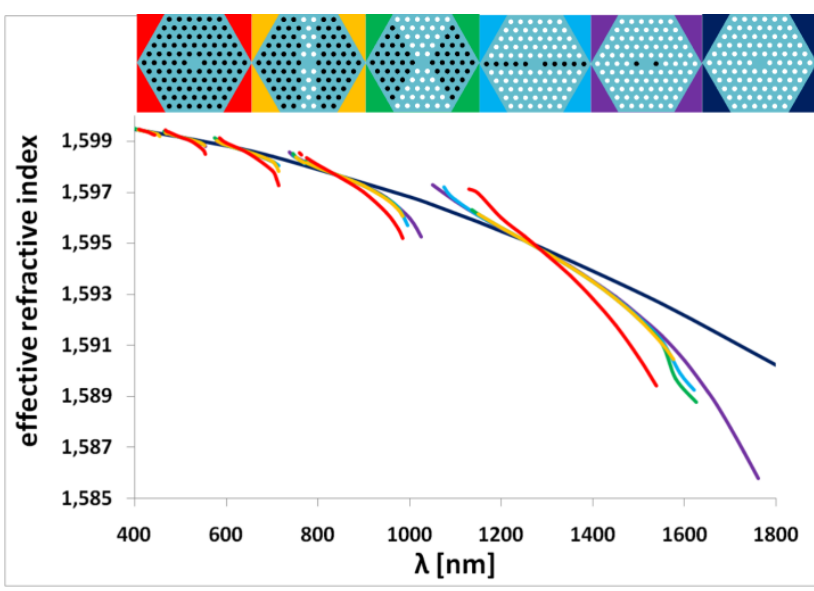

Fig. 3. Effective refractive index for $y$-polarization in investigated structures.

Modal fields in the investigated structures were compared. An exemplary comparison (at wavelength $1550 \mathrm{~nm}$ ) of $\mathrm{x}$-polarized modes is shown in Fig. 2. The profiles of y-polarized modes in each case were almost the same.

The fields of selectively filled structures indicate that confinement losses increase as the number of filled holes increases. It is worth noticing that modal fields calculated for structures "d" and "e" are almost identical.

The effective refractive indices of guided modes decrease with an increasing wavelength within each bandgap (Fig. 3). The changes of a refractive index are greater in structures with a larger number of filled holes. For the selectively filled structures, at some wavelengths (especially at the central part of the bandgap), the effective indices are almost equal, probably as a consequence of the fact that six holes closest to the core are filled in the same way in all four cases (only two opposite holes are filled, Fig. 1b-e).

Phase birefringence was calculated and shown in Fig. 4. The birefringence of an empty structure is zero for all wavelengths, whereas for selectively filled fibers the phase birefringence strongly depends on the wavelength. Within each bandgap, the birefringence decreases with an increasing wavelength. In a uniformly filled structure, the birefringence differs slightly from zero at the ends of bandgaps, but it is probably a numerical error rather than a real physical phenomenon. Surprisingly, in selectively filled structures, the birefringence changes its sign near the red end of each bandgap.

The values of birefringence of selectively filled structures (Fig. 1b-e) for a given wavelength are similar, which can be again explained by the identical filling of six most inner holes. In general, the birefringence in structures " $b$ " and "c" is slightly larger than in "d" and "e" (Fig. 4). More significant differences can be noticed at the edges of the bandgaps.

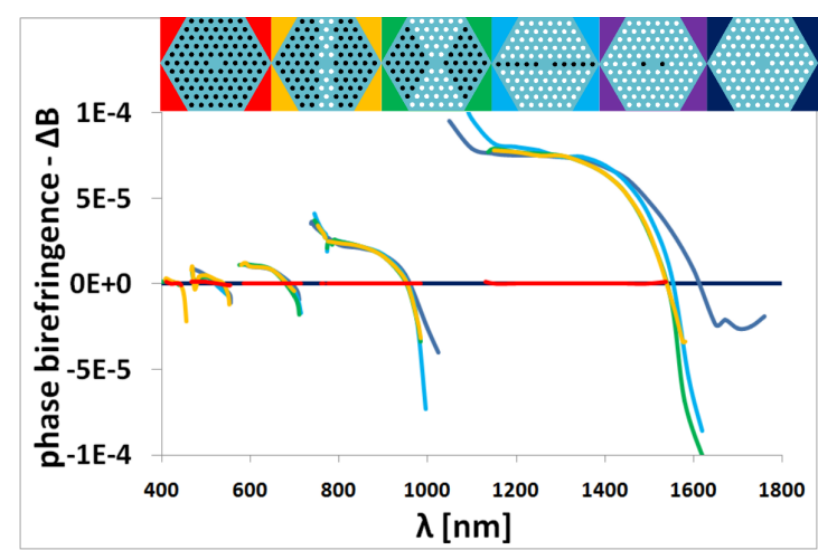

Fig. 4. Phase birefringence in investigated structures.

Confinement losses were calculated from the imaginary part of effective refractive indices of guided modes using the formula below [7].

$$
\alpha=\frac{2 \pi}{\lambda} \frac{20}{\ln 10} \operatorname{Im}\left(n_{\text {eff }}\right)
$$


It must be mentioned that in our calculations the material losses of fiber and liquid have not been taken into account, whereas in real structures filled with liquids the guiding properties could be affected by the attenuation of materials.

Fig. 5 shows the calculated confinement losses of ypolarization. The losses in structure " $b$ " are almost as low as losses in an empty structure. The largest losses occur for a fully filled structure. The losses in structures " $d$ " and "e" have very close values, which is similar to the behavior of birefringence in these structures.

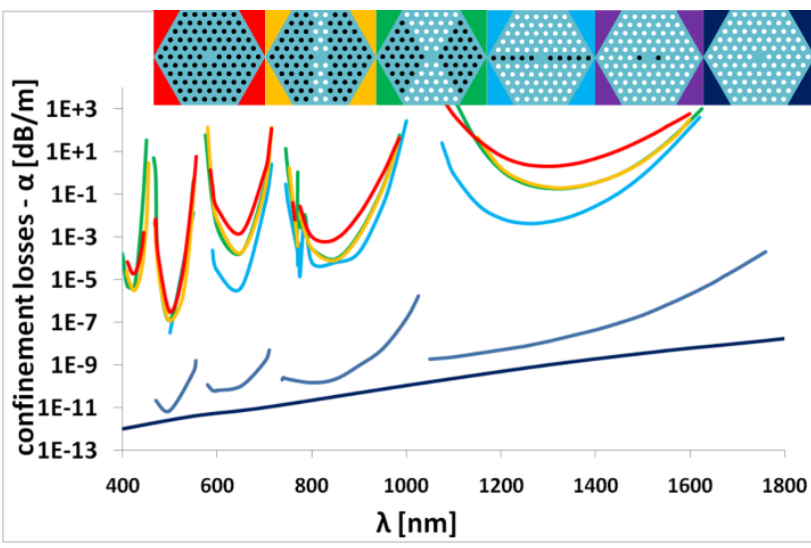

Fig. 5. Confinement losses of y-polarization in investigated structures.

The losses for x-polarization behave in a similar way, which can be illustrated by the polarization-dependent losses in Fig. 6.

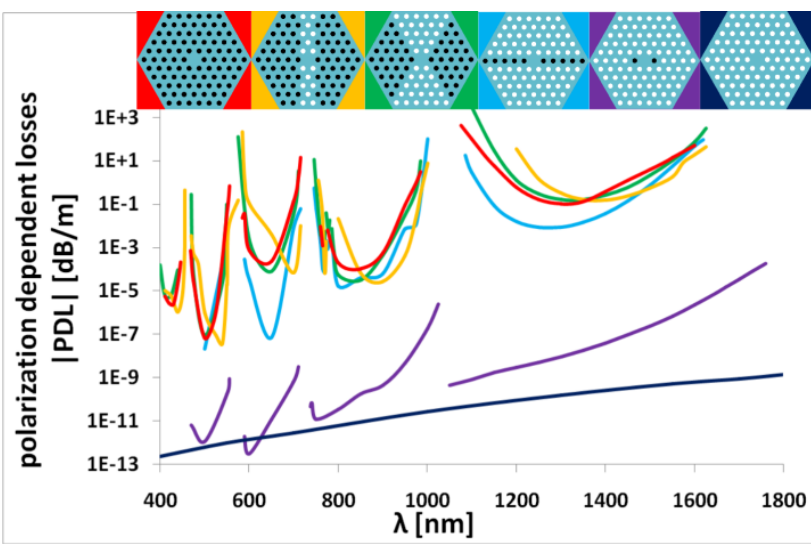

Fig. 6. Polarization dependent losses in investigated structures.

In the figures above, it can be seen that the bandgaps in the investigated structures are similar, but their width depends on the amount of filled holes. The bandgap widths for investigated structures are shown in Fig. 7.

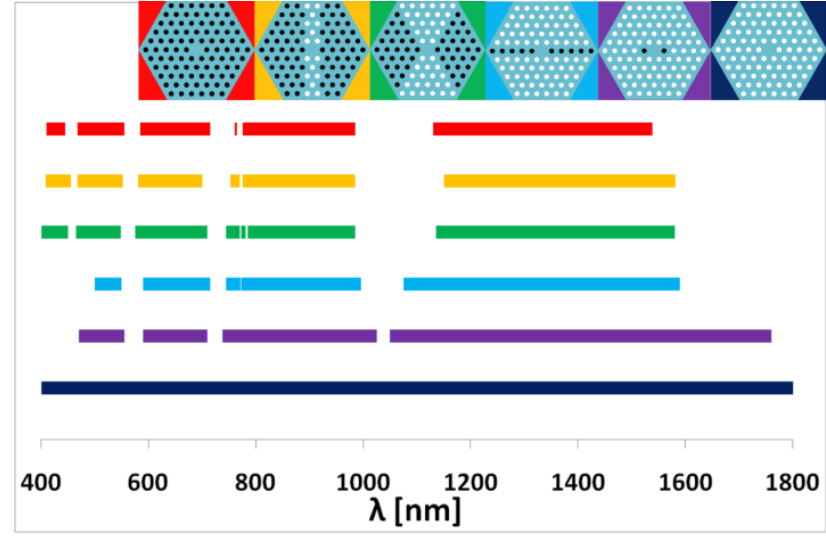

Fig. 7. Scheme of bandgaps widths in investigated structures.

In this letter, numerical modeling of selectively filled photonic crystal fibers was shortly reported. Because the refractive index of the filling material was larger than the index of glass, a photonic bandgap effect was observed. In structures with a large number of filled holes the refractive index decreases more rapidly with an increasing wavelength. The birefringence of selectively filled structures is similar within each bandgap. The higher the number of the filled holes, the larger confinement losses in the fiber structure. The width and position of bandgaps depend on the number of filled holes.

Quite an interesting effect was noticed analyzing the changes of birefringence in selectively filled fibers. It appeared that at some wavelengths (near the red end of each bandgap) the phase birefringence changes its sign. It means that in a selectively filled fiber it is possible to find a few wavelengths at which the birefringence is equal to zero, even if the geometry is obviously suggesting that the fiber should be highly birefringent.

\section{References}

[1] Ph. St. J. Russell, J. Lightwave Technol. 24, 4729 (2006).

[2] Y. Han, S. Tan, M.K. K. Oo, D. Pristinski, S. Sukhishvili, H. Du, Adv. Mat. 22, 2647 (2010.)

[3] S. Ertman, A.H. Rodríguez, M.M. Tefelska, M.S. Chychłowski, D. Pysz, R. Buczyński, E. Nowinowski-Kruszelnicki, R. Dąbrowski, T.R. Woliński, J. Lightwave Technol. 30, 1208 (2012).

[4] K. Mileńko, S. Ertman, T.R. Woliński, Mol. Cryst. Liq. Cryst. 596, 4 (2014).

[5] Y. Wang, C. Liao, X. Zhong, Z. Li, Y. Liu, J. Zhou, K. Yang, Proc. SPIE 8914, 89140J-1 (2013).

[6] F. Wang, W. Yuan, O. Hansen, O. Bang, Opt. Expr. 19, 17585 (2011).

[7] T. Martynkien, G. Statkiewicz-Barabach, J. Olszewski, et al., Opt. Expr. 18, 15113 (2010).

[8] B.T. Kuhlmey, R.C. McPhedran, C.M. de Sterke, Opt. Lett. 27, 1684 (2002) 